\title{
Análisis estructural de la Escala de Bienestar Psicológico de Ryff en universitarios mexicanos
}

\author{
Structural analysis of the Ryff's Psychological Well-Being Scale among \\ Mexican college students
}

\author{
Sergio Dominguez-Lara ${ }^{\mathrm{a}}$, Tania Romo-González ${ }^{\mathrm{b}}$, Carolina Palmeros-Exsome ${ }^{\mathrm{b}}$, \\ Antonia Barranca-Enríquez ${ }^{\mathrm{b}}$, Eduardo del Moral-Trinidad ${ }^{\mathrm{b}}$, Yolanda Campos-Uscanga ${ }^{\mathrm{b}}$ * \\ aUniversidad de San Martín de Porres, Lima, Perú \\ bUniversidad Veracruzana, Xalapa, Veracruz, México
}

\begin{abstract}
Resumen
Objetivos: realizar el análisis de la estructura interna de la versión en español de la Escala de Bienestar Psicológico de Ryff (PWBS-E) en estudiantes universitarios ( $n=1700 ; 54.75 \%$ mujeres; $M_{\text {edad }}=19.23$ ) de una institución pública en Veracruz, México. Método: La estructura interna de la PWBS-E fue evaluada mediante el análisis factorial confirmatorio (CFA), el modelamiento de ecuaciones estructurales exploratorio (ESEM) y el análisis factorial exploratorio (EFA). La confiabilidad del constructo fue estimada con el coeficiente $\alpha$ y de las puntuaciones con el coeficiente $\alpha$. Resultados: indicaron que una estructura unidimensional y breve (19 ítems) posee mayor respaldo empírico. Además, se evidenció la existencia de un factor de método asociado a ítems invertidos en los modelos multidimensionales. En cuanto a la confiabilidad, se hallaron indicadores aceptables en ambos niveles (constructo y puntuaciones). Conclusiones: se discuten los hallazgos y las implicancias prácticas de la versión breve de la PWBS-E en universitarios.
\end{abstract}

Palabras clave: validez, confiabilidad, ESEM, PWBS, bienestar, universitarios.

Para citar este artículo:

Dominguez-Lara, S., Romo-González, T., Palmeros-Exsome, C., BarrancaEnríquez, A., del Moral-Trinidad, E., \& Campos-Uscanga, Y. (2019). Análisis estructural de la Escala de Bienestar Psicológico de Ryff en universitarios mexicanos. Liberabit, 25(2), 267-285. https://doi.org/ 10.24265/liberabit.2019.v25n2.09

\begin{abstract}
Objectives: This paper aimed to analyze the internal structure of the Spanish version of the Ryff's psychological well-being scale (PWBS-E) among college students ( $n=1700,54.75 \%$ females, $\left.M_{\text {age }}=19.23\right)$ who attend a public institution located in Veracruz, Mexico. Method: The internal structure of the PWBS-E was assessed using the confirmatory factor analysis (CFA), exploratory structural equation modeling (ESEM) and exploratory factor analysis (EFA), while the reliability of the construct was estimated with the ù coefficient and the reliability of the scores, with the $\alpha$ coefficient. Results: Suggested that a short and unidimensional structure (19 items) provides greater empirical support. In addition, a method factor associated with inverted items in multidimensional models was evidenced. Regarding the reliability, acceptable indicators were found in both levels (construct and scores). Conclusions: This paper addresses both the findings and practical implications of the short version of the PWBS-E among college students.
\end{abstract}

Keywords: validity, reliability, ESEM, PWBS, wellbeing, college students.

Este es un artículo Open Access bajo la licencia Creative Commons Atribución-NoComercial-CompartirIgual 4.0

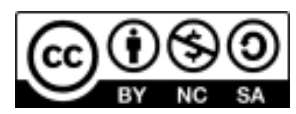




\section{Introducción}

En los últimos años hay un creciente interés por el estudio del bienestar psicológico (BP) con base en la evidencia de su influencia en la salud mental y la presencia de enfermedades crónicas no trasmisibles (Ryff, 2014). El BP hace referencia al sentimiento de autorrealización y al fomento del desarrollo del máximo potencial de las personas, y se trata de la evaluación cognitiva y afectiva que realizan de sus vidas (Ryff, 1989). Carol Ryff desarrolló un modelo para su estudio que incluye seis dimensiones: Autoaceptación (AA), referida a la valoración que hace el individuo de sí mismo; Relaciones positivas con los otros (RPO), relacionada con la calidad de las interacciones que la persona tiene con los demás; Autonomía (AU), vinculada a la toma de decisiones de forma independiente; Dominio del entorno (DE), que involucra la forma en la que la persona utiliza el entorno como fuente de oportunidades; Propósito en la vida (PV), que se orienta a la creencia de que se tienen objetivos o propósitos en la vida; y Crecimiento personal (CP), que hace referencia al grado en que una persona valora su desarrollo y el de sus capacidades (Rosa-Rodríguez, Negrón-Cartagena, Maldonado-Peña, Quiñones-Berrios, \& ToledoOsorio, 2015), las cuales son evaluadas por la Psychological Well-being Scales (Ryff \& Keyes, 1995). La PWBS ha tenido amplia aceptación por parte de la comunidad científica, así como aplicaciones en diversas esferas de la conducta humana (ver Ryff, 2014), destacando el ámbito de la salud.

Por ejemplo, en un estudio que empleó la PWBS, encontró que las medias de BP en personas con obesidad fueron inferiores a las observadas en personas con peso normal, con tamaños del efecto moderados para las dimensiones de autonomía $(d=$ 1.61) y dominio del ambiente $(d=1.88)$ (Magallares, Benito de Valle, Irles, Bolaños-Ríos, \& JaureguiLobera, 2014). Asimismo, desde el lado de la salud física, se ha observado que en comparación con las personas con bajos niveles de BP, las que presentan valores medios y altos tienen mejor salud cardiovascular ( $p=.002$ ) (Boehm et al., 2017); y desde la salud mental, el éxito en la prevención y el tratamiento de depresión, ansiedad y trastornos de la conducta alimentaria están relacionados con el BP (Edmondson \& MacLeod, 2015; Tomba, Offidani, Tecuta, Schumann, \& Ballardini, 2014).

Estos avances con respecto al vínculo entre el BP y la salud se han aplicado al ámbito universitario. Se ha encontrado que mayores niveles de BP aumentan el manejo del estrés, así como la adopción de estilos de vida saludables (Ridner, Newton, Staten, Crawford, \& Hall, 2016), asociándose a su vez con menos depresión y ansiedad (Aboalshamat, Hou, \& Strodl, 2015; Bhullar, Hine, \& Phillips, 2014).

Debido a la posible relación entre el BP y la salud de los estudiantes universitarios (Schnettler et al., 2015), su estudio es prioritario en este grupo poblacional que si bien se encuentra en una etapa clave para la consolidación de estilos de vida y el logro de más altos niveles de bienestar, aún debe lidiar con estresores cotidianos dentro y fuera del ámbito universitario (Dominguez-Lara \& Merino-Soto, 2019). Por tal motivo, se requiere del estudio psicométrico de los instrumentos de los que se dispone en México para que midan de forma precisa el constructo, debido a la importancia de la vida emocional del individuo.

Como se precisó inicialmente, la PWBS es uno de los instrumentos más usados para evaluar el BP, y pese a la existencia de estudios psicométricos que indican que la estructura original de seis factores se recupera de forma satisfactoria (Dierendonck, Díaz, Rodríguez-Carvajal, Blanco, \& Moreno-Jiménez, 2008), otros trabajos brindan configuraciones alternativas como de cuatro (Tomás, Sancho, Melendez, \& Mayordomo, 2012) o cinco factores (Kafka \& Kozma 2002).

La PWBS presenta una versión en español (PWBS-E; Díaz et al., 2006) que posee numerosos estudios instrumentales desarrollados en países latinoamericanos que defienden el modelo de seis 
factores (e.g., Gallardo-Cuadra \& Moyano-Díaz, 2012; Reyna, Delfino, \& Mele, 2013; Véliz-Burgos, 2012), pero en un trabajo de revisión de estos (Dominguez-Lara \& Navarro-Loli, 2018) se concluyó que en el caso de aquellos estudios que basan sus conclusiones en análisis factoriales exploratorios algunos procedimientos ejecutados no eran los apropiados para el estado actual de la investigación psicométrica, como el Little Jiffy (e.g., Reyna et al., 2013). Asimismo, con relación a los análisis confirmatorios, en numerosas ocasiones no fueron interpretadas apropiadamente las elevadas correlaciones interfactoriales (e.g., Dierendonck et al., 2008), que son indicios de redundancia y complejidad factorial; asimismo, no fue analizado el potencial efecto del método asociado a ítems invertidos (e.g., Tomás, Meléndez, Oliver, Navarro-Pardo, \& Zaragoza, 2010); y son escasos aquellos estudios que concluyen que los datos no se ajustan al modelo teórico original de seis factores cuando la evidencia así lo indica (e.g., Chitgian-Urzúa, Urzúa, \& VeraVillarroel, 2013).

En este orden de ideas, existen algunos estudios instrumentales de la PWBS de 39 ítems procedentes de México. Por ejemplo, Medina-Calvillo, GutiérrezHernández y Padrós-Blázquez (2013) analizaron la escala desde un enfoque confirmatorio, pero omitieron el reporte de las cargas factoriales y de las correlaciones interfactoriales, lo que habría permitido una discusión mayor sobre los resultados obtenidos. Por otro lado, en el estudio exploratorio de LoeraMalvaez, Balcázar-Nava, Trejo-González, GurrolaPeña y Bonilla-Muñoz (2008) ejecutado en adultos mexicanos, fue obtenida una estructura más simple por medio de la eliminación de ítems con baja carga factorial; sin embargo, el procedimiento usado fue el Little Jiffy, el cual no es recomendado para diseños instrumentales debido a que el análisis de componentes principales no diferencia varianza común y específica, la determinación del número de factores por medio de la magnitud de los valores propios (o Eigen) tiende a sobreestimar el número de factores a extraer, y la rotación varimax fuerza la ortogonalidad, pese a que las dimensiones de la PWBS están teóricamente relacionadas (ver LloretSegura, Ferreres-Traver, Hernández-Baeza, \& TomásMarco, 2014; Watkins, 2018).

Recientemente la PWBS fue analizada por Balcázar, Esparza y Gurrola (2018) quienes concluyen a favor de una estructura de tres dimensiones bajo un enfoque confirmatorio en universitarios mexicanos, pero existen algunos aspectos a considerar. En primer lugar, el análisis inicial fue exploratorio, a pesar de la existencia de una estructura original ya definida de seis factores y que podría evaluarse mediante un análisis confirmatorio. En segundo lugar, en el análisis exploratorio no fue usado (o al menos no se reportó) un método para determinar qué número de factores se debe extraer. En tercer lugar, se indica que la solución de tres factores es la opción más viable, aunque sin analizar la complejidad factorial de los ítems, la cual en algunos casos llega a ser evidente (e.g., el ítem 1 tiene cargas de .57, -.28 y .29 en los tres factores). Por último, reportan que las correlaciones entre puntuaciones que son de magnitud moderada pero las correlaciones interfactoriales, que tienden a ser de mayor magnitud, no son informadas, por lo que es probable que exista superposición de factores.

En vista de lo expuesto, el nuevo estudio psicométrico con universitarios mexicanos se justifica metodológicamente en la medida que pretende superar algunas falencias y lagunas de información observadas en los reportes anteriores, como la secuencia en cuanto al empleo de un enfoque analítico-factorial cada vez menos restrictivo o brindar información que pueda ayudar a un mejor entendimiento de la estructura interna de la PWBS. De este modo, para el análisis de la estructura interna se partió de un enfoque analítico-factorial más restrictivo (análisis factorial confirmatorio; CFA) al evaluar los modelos de medición existentes en la literatura (4 a 6 factores; Kafka \& Kozma 2002; Ryff \& Keyes, 1995; Tomás et al., 2012); y si con la primera opción (CFA) no se obtiene evidencia favorable, se implementan métodos 
menos restrictivos como el modelamiento exploratorio de ecuaciones estructurales (ESEM), y finalmente el análisis factorial exploratorio (EFA) a fin de determinar qué estructura factorial explica mejor la variabilidad de los ítems.

Por tales motivos, contar con evidencias de validez en estudiantes universitarios permitirá hacer evaluaciones más precisas para identificar necesidades de intervención y, con el tiempo, favorecer el desarrollo de adecuados niveles de BP. La investigación en este tema se verá también ampliamente fortalecida al contar con una escala con evidencias de validez para su estudio que permita explicar de mejor manera las asociaciones que puede tener con estilos de vida, dinámica familiar, transición entre distintas etapas de la vida, personalidad, trabajo $\mathrm{y}$ algunos componentes del estado de salud.

En ese sentido, el objetivo del presente trabajo fue analizar la estructura interna y confiabilidad de la PWBS-E desde diversos enfoques analítico-factoriales en estudiantes universitarios mexicanos.

\section{Método}

\section{Diseño}

Se realizó un estudio instrumental (Ato, López, \& Benavente, 2013), orientado a la evaluación de las propiedades psicométricas, validez y fiabilidad, de la versión en español de la PWBS.

\section{Participantes}

Fue evaluada una muestra de 1700 estudiantes de pregrado de una universidad pública mexicana ubicada en el estado de Veracruz (54.75\% mujeres), de un rango de edad entre 17 a 48 años $\left(M_{\text {edad }}=19.23\right.$; rango 17 - 25 años $=99.977 \%)$. Este grupo se dividió aleatoriamente en dos muestras no probabilísticas, una para la calibración y otra para la replicación.

La muestra de calibración estuvo integrada por 876 estudiantes (54\% mujeres) con edad promedio de
19.19 años $\left(D E_{\text {edad }}=2.26\right)$. La muestra de replicación estuvo integrada por 824 estudiantes (55.5\% mujeres; $M_{\text {edad }}=19.26$ años; $\left.D E_{\text {edad }}=2.37\right)$. Se incluyeron estudiantes inscritos en el periodo agosto - diciembre 2017 que aceptaron participar en el estudio y no presentaron alguna limitación para realizar el autollenado del instrumento en forma impresa convencional. En ambas muestras se incluyeron estudiantes de todas las áreas académicas que se ofertan en la región Veracruz: ciencias biológicoagropecuarias, ciencias de la salud, económicoadministrativas, humanidades y técnica. Por último, no hubo diferencias en cuanto a la distribución según sexo $\left(\chi^{2}=.368, p=.544\right)$ ni edad $\left(t_{[1658]}=-.574, p\right.$ $=.566)$.

\section{Instrumento}

La ficha de datos sociodemográficos contempló aspectos como sexo, edad y licenciatura. Adicionalmente, en vista de que esta aplicación forma parte de un estudio que toma en cuenta aspectos antropométricos, también se tomó nota del peso, talla, circunferencia de la cintura y porcentaje de grasa corporal.

Se empleó la versión de la Escala de Bienestar Psicológico de Ryff propuesta por Dierendonck (2004) y adaptada al español (PWBS-E; Díaz et al., 2006). Este autoinforme consta de 39 ítems con formato de respuesta tipo Likert que va de 1 (muy en desacuerdo) a 6 (muy de acuerdo). Tiene seis dimensiones: Autoaceptación (6 ítems), Autonomía (8 ítems), Crecimiento personal (7 ítems), Dominio del entorno (6 ítems), Propósito en la vida (6 ítems) y Relaciones positivas con los otros (6 ítems).

\section{Procedimiento}

Inicialmente, la comprensión de la escala fue analizada solicitando la opinión de 40 estudiantes universitarios mexicanos (20 hombres y 20 mujeres) con las mismas características de la muestra evaluada, quienes fueron consultados en grupos pequeños (4 - 
6 personas), ya que en estudios previos se utilizó la versión española, pero sin examinar si los ítems eran comprensibles por los estudiantes mexicanos Se identificó que todos los ítems eran comprensibles por lo que se procedió a su aplicación. Previo a la administración de instrumentos, se invitó a los estudiantes a participar en la investigación y a quienes aceptaron se les proporcionó el consentimiento informado y un compilado de instrumentos, entre los que estaba la PWBS-E, como parte de un proyecto de mayor envergadura liderado por la autora de correspondencia.

Las evaluaciones fueron realizadas de septiembre a diciembre de 2017. Los participantes contestaron de forma individual una versión impresa de la escala de Ryff, en un tiempo promedio de respuesta de 8 minutos. Los estudiantes destinaron en total alrededor de 40 minutos a la respuesta de todas las escalas

\section{Análisis de datos}

Una vez elaborada la base de datos, se detectó que el porcentaje de casos perdidos fue bastante bajo (solo 2 celdas vacías); mucho menor que el 5\% considerado mínimamente problemático (FernándezAlonso, Suárez-Álvarez, \& Muñiz, 2012). Por ello, los datos faltantes fueron imputados con la moda (Shrive, Stuart, Quan, \& Ghali, 2006). De forma previa al análisis estructural, fue evaluada la aproximación a la normalidad univariada de cada ítem por medio de la magnitud de su asimetría y curtosis, esperando valores menores a 3 y 10 , respectivamente (Kline, 2016). Además, se calculó del índice estandarizado de asimetría (IEA; Malgady, 2007), donde magnitudes entre .25 y .50 refieren un acercamiento a la normalidad univariada. La normalidad multivariada fue evaluada con el coeficiente de Mardia (1970) esperando magnitudes por debajo de 70 (Rodríguez \& Ruiz, 2008).
En ese sentido, con la muestra de calibración se ejecutó un CFA y luego de este un modelamiento exploratorio de ecuaciones estructurales (ESEM), ambos con el programa Mplus versión 7 (Muthén \& Muthén, 1998-2015) y con el método de estimación WLSMV (Weighted Least Squares Mean and Variance Adjusted) y correlaciones policóricas.

En cuanto al CFA, fueron analizados tres modelos: de cuatro $\left(\mathrm{M}_{1}\right.$; Tomás et al., 2012), de cinco $\left(\mathrm{M}_{2}\right.$; Kafka \& Kozma 2002) y de seis factores ( $\mathrm{M}_{3}$; Ryff \& Keyes, 1995), y en cada uno de ellos se evaluó la cantidad de malas especificaciones asociadas a cargas cruzadas (Saris, Satorra, \& Veld, 2009) con base en índices de modificación (IM) estadísticamente significativos $\left(\chi^{2}>10\right)$ con un módulo especializado (Dominguez-Lara, \& Merino-Soto, 2018). Una mala especificación hace referencia a dos situaciones: sobreparametrización (cuando se especifica un parámetro cuyo valor poblacional es cero) e infra-parametrización (cuando no se especifica un parámetro cuyo valor poblacional es diferente de cero) (Hu \& Bentler, 1998).

Además, se esperó que la raíz cuadrada de la varianza media extraída ( $\sqrt{ } \mathrm{VME})$ de cada factor sea mayor que la correlación interfactorial $(\phi)$, lo que permite la diferenciación entre factores (Fornell \& Larcker, 1981). La VME es el promedio de las comunalidades de los ítems en determinado factor.

Adicionalmente, se evaluó un modelo de siete factores, seis factores sustantivos y un factor de método (FM) asociado a los ítems invertidos $\left(\mathrm{M}_{4}\right)$, y su impacto sobre la estructura de la PWBS se determinó comparando la VME de los ítems invertidos dentro de cada factor sustantivo antes y después de la inclusión del FM. 


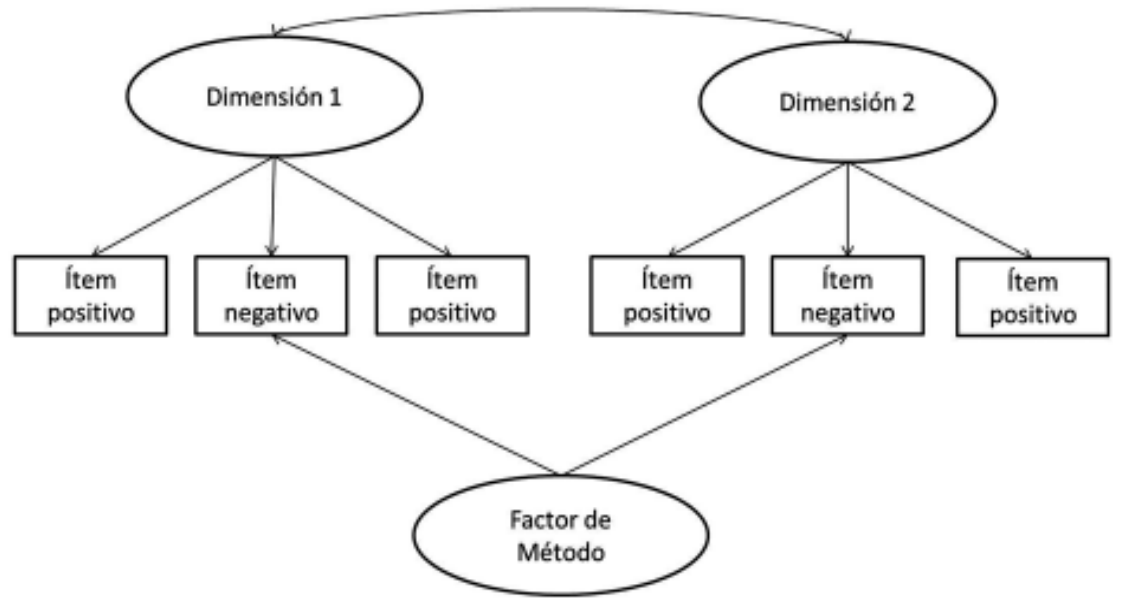

Figura 1. Ejemplo de modelo confirmatorio usado para evaluar los efectos de método asociados a ítems invertidos.

Entonces, a fin de cuantificar la importancia de la presencia del FM se propone el porcentaje de atenuación por presencia del FM (\%FM):

$$
\% F M=\left(\frac{V M E-V M E_{F M}}{V M E}\right)
$$

Donde VME es la varianza media extraída de los ítems invertidos antes de incluir el FM, y $\mathrm{VME}_{\mathrm{FM}}$ es la varianza media extraída de los ítems invertidos luego de incluir el FM. El indicador permitirá saber qué proporción de varianza pierde el factor original en presencia del factor de método.

En el caso del ESEM, además del método de extracción WLSMV, se especificó la rotación geomin ( $\varepsilon=.05$; Asparouhov \& Muthen, 2009). Se evaluaron de forma consecutiva modelos de uno hasta seis factores, incluyendo uno de siete factores donde se espera que el séptimo factor represente al FM de forma similar al AFC. La relevancia de las cargas secundarias se evaluó con el índice de simplicidad factorial (ISF; Fleming \& Merino, 2005), donde valores $>.70$ representan una simplicidad aceptable, es decir que el ítem recibe influencia significativa predominantemente de un factor.
Previo al EFA se determinó el número de factores que podrían extraerse mediante el análisis paralelo (Timmerman \& Lorenzo-Seva, 2011). Posteriormente, y una vez verificada la viabilidad de los datos para ejecutar el EFA con el análisis del KMO y el test de esfericidad de Bartlett, los datos se analizaron con el método de extracción Robust Diagonally Weighted Least Squares (RDWLS; Yang-Wallentin, Jöreskog, \& Luo, 2010) en vista de las características distribucionales de los ítems usando el programa FACTOR (Lorenzo-Seva \& Ferrando, 2013).

Para valorar el ajuste del modelo en todos los análisis (CFA, ESEM y EFA) fueron considerados el índice de ajuste comparativo (CFI > .90; McDonald \& Ho, 2002), el índice de aproximación de la raíz de cuadrados medios del error (RMSEA) interpretado tanto de forma puntual (< .08; Jöreskog \& Sörbom, 1993) como en función al límite superior de su intervalo de confianza $(<.10$; West, Taylor, \& Wu, 2012), y la raíz cuadrada media residual ponderada (WRMR < 1; DiStefano, Liu, Jiang, \& Shi, 2018; Yu \& Muthen, 2002).

Posteriormente, el mejor modelo de medición hallado con la muestra de calibración fue evaluado en la muestra de replicación. 
La equivalencia del modelo unidimensional entre las muestras de calibración y de replicación fue comprobada mediante un análisis de invarianza de medición (Pendergast, Embse, Kilgus, \& Eklund, 2017): invarianza configural, métrica y fuerte. Es necesario mencionar que no se evaluó la igualdad entre residuales (invarianza estricta) porque no es el objetivo del presente estudio (Byrne, 2008). El grado de invarianza alcanzado fue valorado por medio de la variación en la magnitud de los índices de ajuste entre modelos, específicamente del CFI y RMSEA: rechazar la invarianza de medición si $\Delta$ CFI < -.01 y $\triangle$ RMSEA $\geq .01$ (Chen, 2007). Además, de forma complementaria fue considerada la variación en el $\chi^{2}$ entre modelos anidados (Asparouhov \& Muthén, 2006), y una cantidad de parámetros no-invariantes menor que el 20\% (Dimitrov, 2010).

Para terminar, la confiabilidad del constructo fue estimada con el coeficiente $\omega$, esperando magnitudes moderadas (> .70; Hunsley \& Mash, 2008). La confiabilidad de las puntuaciones fue evaluada con el coeficiente $\alpha$ (Cronbach, 1951).

\section{Resultados}

\section{Análisis descriptivo}

En la mayoría de los casos la asimetría y curtosis estuvo en el rango esperado. Sin embargo, los IEA de los ítems de algunos factores originales como Crecimiento personal y Propósito en la vida mostraron valores por encima de lo esperado (> .50; Tabla 1), lo que informa de un alejamiento leve de la normalidad, reforzando así la decisión de usar matrices policóricas en los análisis factoriales.

\section{Evidencias de validez basadas en la estructura interna}

El análisis de los modelos de medición fue realizado de forma secuencial con la muestra de calibración, considerando en primer lugar aquellas configuraciones provistas por la literatura disponible (de 4 a 6 factores). Inicialmente fueron evaluados los modelos de cuatro y cinco factores, los cuales además del incumplimiento de la normalidad multivariada ( Mardia $_{4 \text {-factores }}=$ 233.270; Mardia $_{\text {5-factores }}=416.651 ;$ Mardia $_{\text {6-factores }}=$ 416.651) presentaron índices de ajuste insuficientes y correlaciones interfactoriales que, incluso, llegaban a superar la unidad y eran mayores que la «VME de las dimensiones (Tabla 2). Del mismo modo, el modelo original de seis factores no tuvo respaldo estadístico: índices de ajuste por debajo del límite esperado, correlaciones interfactoriales de alta magnitud, y una cantidad significativa de malas especificaciones asociadas a cargas cruzadas (carga factorial significativa en un factor distinto al original), es decir que no se especificaron cargas cruzadas que podrían ser relevantes al modelo. Sin embargo, una vez que se agregó un factor de método (FM) asociado a los ítems invertidos, los índices de ajuste estadístico mejoraron de forma sustancial y la cantidad de malas especificaciones disminuyó, aunque las correlaciones interfactoriales se mantuvieron por encima de lo permitido y el WRMR sigue siendo elevado (Tabla 2).

Un análisis pormenorizado de los ítems invertidos indica que en presencia del FM disminuye la VME, llegando a atenuarse en más del $50 \%$ en la mayoría de los casos (Tabla 3), es decir que la proporción de varianza de los ítems explicada por el factor teórico se reduce. De ello, se infiere que los ítems invertidos se asocian en mayor grado al FM que al factor que teóricamente debe evaluar, por lo que la presencia del FM es relevante.

En cuanto al modelamiento ESEM, fueron evaluados diversos modelos: desde un modelo unidimensional hasta uno de seis factores. Las estructuras de uno, dos y tres factores no obtuvieron índices de ajuste adecuados, y, por el contrario, las de cuatro y cinco factores obtuvieron mejores índices de ajuste (Tabla 4). Sin embargo, la distribución de ítems en los factores no fue consistente con la estructura teórica del instrumento, es decir que el agrupamiento de ítems siguió un patrón aleatorio y con ítems complejos. 
Tabla 1

Estadísticos descriptivos de los ítems de la PWBS-E: muestra de calibración y replicación

\begin{tabular}{|c|c|c|c|c|c|c|c|c|c|c|}
\hline & \multicolumn{5}{|c|}{ Muestra de calibración } & \multicolumn{5}{|c|}{ Muestra de replicación } \\
\hline & $M$ & $D E$ & $\mathrm{~g}_{1}$ & IEA & $\mathrm{g}_{2}$ & $M$ & $D E$ & $\mathrm{~g}_{1}$ & IEA & $g_{2}$ \\
\hline \multicolumn{11}{|c|}{ F1: Autoaceptación } \\
\hline Ítem 1 & 4.662 & 1.102 & -.896 & .369 & .959 & 4.727 & 1.014 & -.753 & .366 & .761 \\
\hline Ítem 7 & 4.855 & 1.122 & -1.086 & .432 & 1.083 & 4.886 & 1.123 & -1.086 & .431 & 1.137 \\
\hline Ítem 19 & 4.962 & 1.02 & -1.266 & .609 & 1.966 & 4.973 & .971 & -.953 & .506 & .927 \\
\hline Ítem 31 & 5.018 & .998 & -1.122 & .563 & 1.546 & 4.972 & 1.032 & -1.221 & .573 & 1.840 \\
\hline Ítem 13R & 2.752 & 1.403 & .484 & .123 & -.641 & 2.867 & 1.464 & .454 & .106 & -.716 \\
\hline Ítem 25R & 4.269 & 1.511 & -.607 & .133 & -.651 & 4.283 & 1.429 & -.478 & .117 & -.790 \\
\hline \multicolumn{11}{|c|}{ F2: Autonomía } \\
\hline Ítem 3 & 4.685 & 1.245 & -.924 & .298 & .381 & 4.638 & 1.268 & -.891 & .277 & .261 \\
\hline Ítem 10 & 4.491 & 1.393 & -.865 & .223 & -.014 & 4.552 & 1.367 & -1.024 & .274 & .437 \\
\hline Ítem 21 & 4.781 & 1.046 & -.909 & .415 & .950 & 4.784 & 1.049 & -.978 & .444 & 1.025 \\
\hline Ítem 27R & 3.265 & 1.517 & .137 & .030 & -1.050 & 3.234 & 1.518 & .066 & .014 & -1.085 \\
\hline Ítem 4R & 4.155 & 1.554 & -.430 & .089 & -.997 & 4.050 & 1.569 & -.351 & .071 & -1.008 \\
\hline Ítem 9R & 4.304 & 1.558 & -.585 & .121 & -.803 & 4.290 & 1.552 & -.557 & .116 & -.816 \\
\hline Ítem 15R & 3.674 & 1.441 & -.023 & .006 & -.893 & 3.638 & 1.448 & .091 & .022 & -.997 \\
\hline Ítem 33R & 3.857 & 1.434 & -.119 & .029 & -.902 & 3.876 & 1.422 & -.132 & .033 & -.918 \\
\hline \multicolumn{11}{|c|}{ F3: Crecimiento personal } \\
\hline Ítem 24 & 5.350 & .817 & -1.443 & 1.082 & 2.823 & 5.302 & .854 & -1.714 & 1.176 & 4.331 \\
\hline Ítem 35 & 5.138 & .963 & -1.393 & .750 & 2.604 & 5.130 & .946 & -1.391 & .777 & 2.578 \\
\hline Ítem 37 & 4.969 & 1.014 & -1.177 & .572 & 1.656 & 4.911 & 1.02 & -1.109 & .533 & 1.477 \\
\hline Ítem 38 & 5.203 & .878 & -1.302 & .845 & 2.512 & 5.216 & .843 & -1.059 & .744 & 1.044 \\
\hline Ítem 30R & 4.328 & 1.493 & -.673 & 151 & -.601 & 4.341 & 1.441 & -.591 & .142 & -.65 \\
\hline Ítem 34R & 3.767 & 1.440 & -.156 & .038 & -.836 & 3.752 & 1.45 & -.145 & .034 & -.867 \\
\hline Ítem 36R & 3.760 & 1.608 & -.199 & .039 & -1.193 & 3.773 & 1.565 & -.181 & .037 & -1.082 \\
\hline \multicolumn{11}{|c|}{ F4: Dominio de entorno } \\
\hline Ítem 11 & 4.495 & 1.216 & -.879 & .297 & .545 & 4.507 & 1.205 & -.908 & .312 & .520 \\
\hline Ítem 16 & 4.914 & 1.037 & -1.259 & .586 & 2.039 & 4.943 & 1.026 & -1.313 & .624 & 2.183 \\
\hline Ítem 28 & 4.664 & 1.015 & -.613 & .297 & .322 & 4.610 & 1.025 & -.701 & .334 & .574 \\
\hline Ítem 39 & 5.015 & 1.135 & -1.478 & .574 & 2.261 & 4.913 & 1.154 & -1.375 & .516 & 1.896 \\
\hline Ítem 5R & 4.207 & 1.505 & -.510 & .113 & -.826 & 4.172 & 1.484 & -.463 & .105 & -.895 \\
\hline Ítem 22R & 3.970 & 1.458 & -.374 & .088 & -.802 & 3.959 & 1.436 & -.300 & .073 & -.83 \\
\hline \multicolumn{11}{|c|}{ F5: Propósito en la vida } \\
\hline Ítem 6 & 5.037 & 1.044 & -1.355 & .622 & 2.151 & 5.063 & 1.061 & -1.404 & .624 & 2.082 \\
\hline Ítem 12 & 4.857 & .942 & -.821 & .462 & 1.099 & 4.818 & .989 & -.808 & .413 & .661 \\
\hline Ítem 17 & 4.749 & 1.122 & -1.014 & .403 & 1.098 & 4.765 & 1.085 & -.954 & .405 & 1.032 \\
\hline Ítem 18 & 4.865 & 1.041 & -1.057 & .488 & 1.423 & 4.862 & 1.041 & -1.035 & .478 & 1.236 \\
\hline Ítem 23 & 5.033 & 1.032 & -1.323 & .621 & 2.226 & 5.067 & 1.004 & -1.276 & .633 & 2.039 \\
\hline Ítem 29R & 4.565 & 1.466 & -.928 & .216 & -.147 & 4.507 & 1.485 & -.796 & .180 & -.444 \\
\hline \multicolumn{11}{|c|}{ F6: Relaciones positivas con los otros } \\
\hline Ítem 14 & 4.732 & 1.078 & -.808 & .348 & .606 & 4.715 & 1.034 & -.869 & .406 & 1.006 \\
\hline Ítem 32 & 5.002 & 1.076 & -1.239 & .534 & 1.468 & 5.007 & 1.058 & -1.290 & .576 & 1.798 \\
\hline Ítem 2R & 4.114 & 1.547 & -.464 & .097 & -.928 & 4.157 & 1.540 & -.512 & .108 & -.923 \\
\hline Ítem 8 & 4.168 & 1.565 & -.505 & .103 & -.902 & 4.154 & 1.563 & -.483 & .099 & -.914 \\
\hline Ítem 20R & 3.455 & 1.579 & .045 & .009 & -1.117 & 3.536 & 1.590 & .026 & .005 & -1.190 \\
\hline Ítem 26R & 3.812 & 1.552 & -.132 & .027 & -1.122 & 3.871 & 1.546 & -.184 & .038 & -1.136 \\
\hline
\end{tabular}

Nota: $M$ = Media; $D E$ = Desviación estándar; $\mathrm{g}_{1}=$ Asimetría; IEA = Índice estandarizado de asimetría; $\mathrm{g}_{2}=$ Curtosis; en negrita = IEA $\geq .50$ 
Tabla 2

Índices de ajuste de los modelos de medición de la PWBS-E

\begin{tabular}{|c|c|c|c|c|c|c|}
\hline \multirow[b]{2}{*}{ Índices de ajuste } & \multicolumn{3}{|c|}{ Índices de ajuste } & \multicolumn{3}{|c|}{ Bondad del modelo } \\
\hline & CFI & RMSEA (IC 90\%) & WRMR & ${ }{ } \mathrm{ME}_{\text {(rango) }}$ & $\phi_{\text {promedio (mín. - máx.) }}$ & $\mathrm{ME}_{\text {cargas cruzadas }}$ \\
\hline 4 factores 0 & .794 & $.137(.133, .140)$ & 3.204 & $.526-.703$ & $.962(.853-1.055)$ & - \\
\hline 5 factores oblicuos & .777 & $.104(.102, .107)$ & 3.363 & $.533-.703$ & $.736(.551-.974)$ & - \\
\hline 6 factores oblicuos & .779 & $.105(.102, .107)$ & 3.353 & $.525-.703$ & $.797(.551-1.054)$ & 87 de 104 (83.7\%) \\
\hline $\begin{array}{l}\text { factores oblicuos } \mathrm{c} \\
\text { factor de método }\end{array}$ & .925 & $.061(.059, .064)$ & 1.696 & - & $.754(.422-1.040)$ & 34 de $51(66.7 \%)$ \\
\hline
\end{tabular}

Nota: $\phi$ = correlación interfactorial; ME = malas especificaciones.

Tabla 3

Estructura interna y fiabilidad de la PWBS-E: AFC

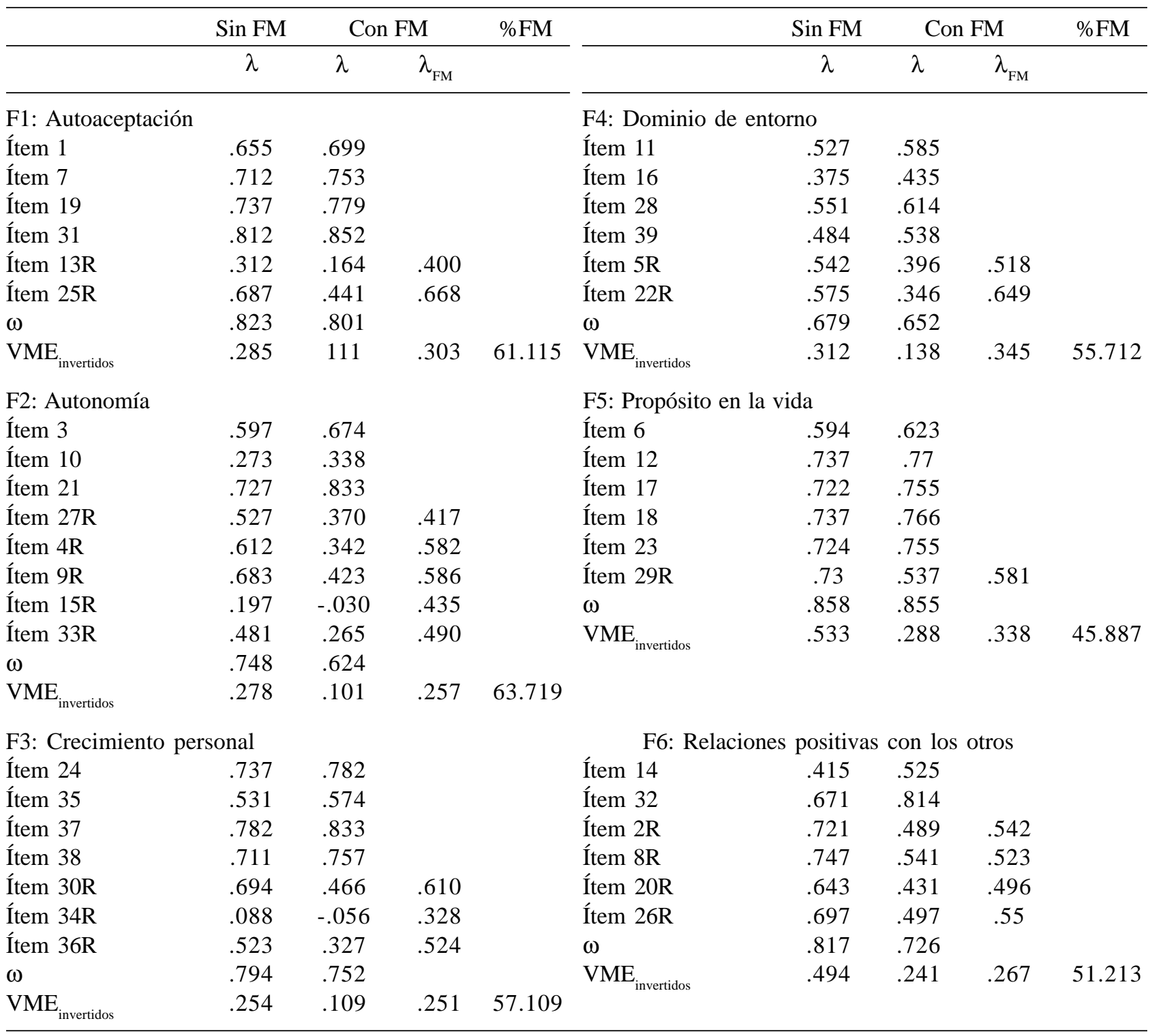

Nota: $\mathrm{R}$ = ítem invertido; VME = Varianza media extraída; \%FM = porcentaje de atenuación de la VME por presencia del FM. 
Tabla 4

Estructura interna y fiabilidad de la PWBS-E: ESEM

\begin{tabular}{lllcc}
\hline \multicolumn{1}{c}{ Índices de ajuste } & CFI & RMSEA (IC 90\%) & WRMR & $\phi_{\text {promedio (mín. - máx.) }}$ \\
\hline 1 factor & .743 & $.111(.109, .114)$ & 3.638 & - \\
2 factores oblicuos & .888 & $.076(.073, .078)$ & 1.909 & .276 \\
3 factores oblicuos & .913 & $.069(.066, .071)$ & 1.605 & .278 \\
4 factores oblicuos & .938 & $.060(.057, .062)$ & 1.264 & .259 \\
5 factores oblicuos & .955 & $.052(.050, .055)$ & 1.057 & .240 \\
6 factores oblicuos & .968 & $.046(.043, .049)$ & .883 & .234 \\
6 factores oblicuos + Factor de método & .975 & $.042(.039, .045)$ & .777 & - \\
& & & & - \\
6 factores sin ítems invertidos & .984 & $.054(.048, .060)$ & .661 & - \\
5 factores sin ítems invertidos & .977 & $.061(.055, .066)$ & .816 & - \\
4 factores sin ítems invertidos & .968 & $.067(.062, .071)$ & .981 & - \\
\hline
\end{tabular}

Nota: $\phi=$ correlación interfactorial.

Por otro lado, el modelo de seis factores obtuvo mejores índices de ajuste, pero la estructura resultante presentó parámetros estadísticos desfavorables. En primer lugar, el agrupamiento de ítems difiere de la estructura original ya que existen cargas factoriales que son muy bajas para ser consideradas significativas en su respectivo factor (e.g., ítem 4 en Autonomía) o, simplemente, el factor no se llega a configurar (e.g., Propósito en la vida). En segundo lugar, aunque ciertos ítems presentan carga factorial aceptable (e.g., ítem 37 en Crecimiento personal), estos son factorialmente complejos (ISF < .70), lo que cuestiona la representatividad del factor teórico que evalúa (Tabla 5). Los índices de ajuste mejoran sustancialmente con la inclusión de un séptimo factor, asociado a los ítems invertidos, aunque las cargas factoriales siguen siendo bajas (e.g., ítem 10 en Autonomía) y continúan existiendo ítems factorialmente complejos (Tabla 5).

En vista de la evidencia desfavorable con modelos más restrictivos (CFA y ESEM), se empleó un EFA solo con los ítems directos, dado que los ítems invertidos resultaron problemáticos.
Previamente se corroboró la adecuación de la matriz de correlaciones (KMO = .937; Estadístico de Bartlett $=7060.3 ; p<.001)$ y con el análisis paralelo se determinó la extracción de un solo factor que explica el $43.037 \%$ de la variabilidad de las puntuaciones. Luego, con el método de estimación Robust Diagonally Weighted Least Squares (RDWLS) con base en matrices policóricas, se hallaron índices de ajuste adecuados (CFI $=.978$, RMSEA $=.060$ $\left.\left[p_{\text {RMSEA }<.05}=1.000\right], \mathrm{WRMR}=.058\right)$. Pese a ello, se detectaron tres ítems con cargas factoriales de magnitud no aceptable $\left(\lambda_{10}=.292 ; \lambda_{14}=.374 ; \lambda_{16}=\right.$ $.478)$, por lo que se decidió retirarlos.

Tras el segundo análisis realizado, el factor extraído explicó el $47.421 \%$ de variabilidad, sus índices de ajuste fueron buenos (CFI $=.982$, RMSEA $=.062$ $\left[p_{\text {RMSEA } .05}=1.000\right]$, WRMR $\left.=.056\right) \mathrm{y}$ las cargas factoriales adecuadas, aunque heterogéneas (de .502 a .809; Tabla 6).

Con la muestra de replicación se realizó un CFA para un solo factor, y el panorama fue aceptable considerando los índices de ajuste (CFI = .922; RMSEA = .099 [IC90\% .094, .104]; WRMR = 1.783), evidenciando además cargas factoriales de magnitud aceptable (ver anexos). 


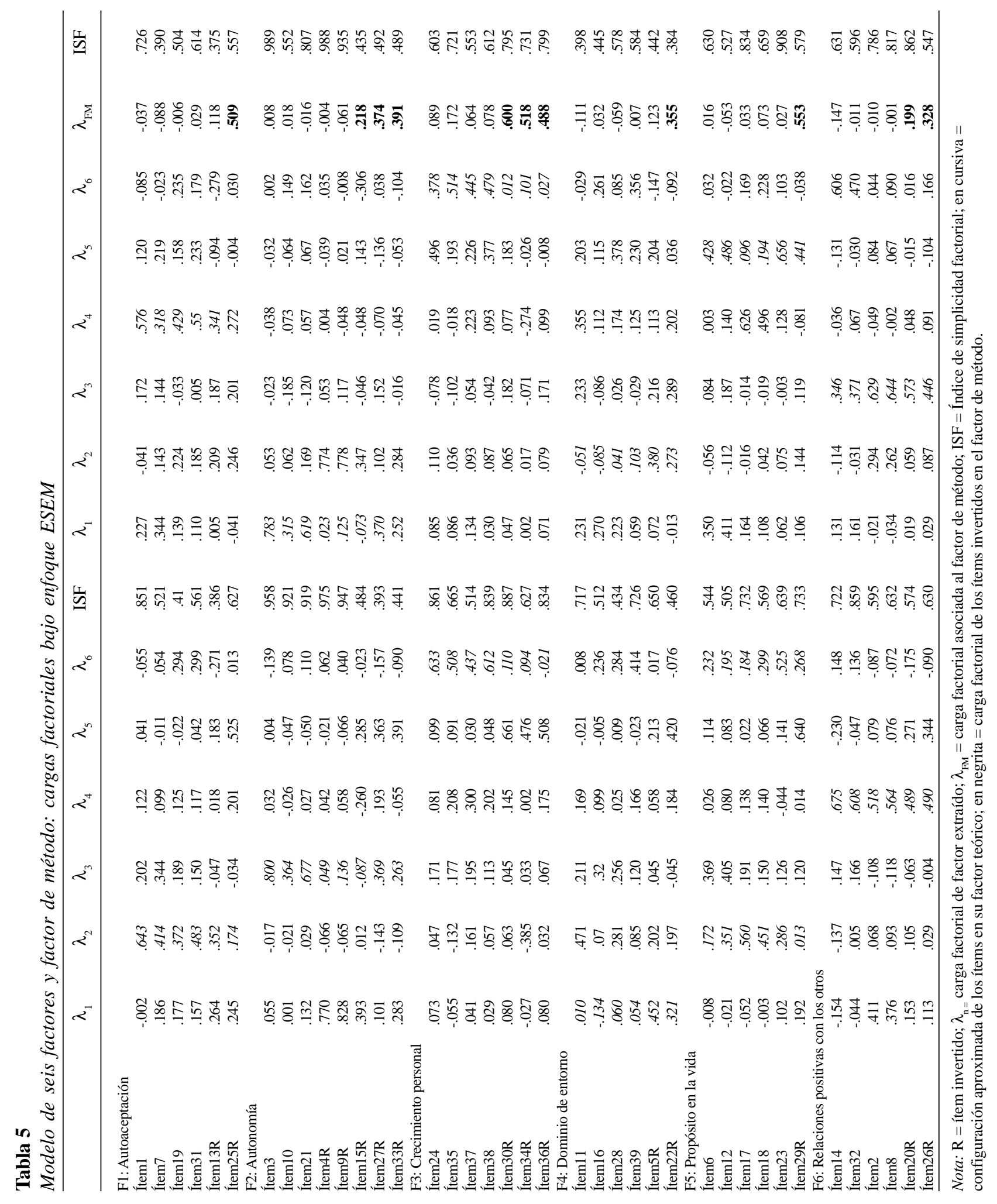




\section{Modelo final: análisis de invarianza entre muestras}

En vista de los resultados conseguidos luego de ejecutar el análisis de invarianza de medición y de valorar globalmente la variación de los índices de ajuste, la diferencia no significativa entre $\operatorname{los} \chi^{2}$ de modelos anidados (ver anexos) -y que solo dos cargas factoriales $\left(\mathrm{IM}_{\text {item } 1}=10.831 ; \mathrm{IM}_{\text {item } 8}=10.303\right)$ fueron no-invariantes cuando se evaluó la invarianza métricase puede considerar que el modelo unidimensional es invariante entre muestras.

\section{Confiabilidad}

En cuanto a la confiabilidad, los coeficientes obtenidos fueron elevados tanto con la muestra de calibración $(\omega=.939 ; \alpha=.910)$ como de replicación $(\omega=.937 ; \alpha=.909)$.

\section{Discusión}

El estudio de las propiedades métricas de los instrumentos de evaluación psicológica es una tarea continua, la cual inevitablemente está sujeta al avance y complejidad de los métodos, aplicaciones contemporáneas en psicometría y medición psicológica, así como a la utilidad e impacto de la medida en cuestión. En ese sentido, es obligatorio reanalizar instrumentos que, si bien son de uso masivo e incluso mundial, podrían presentar algunas falencias o limitaciones a la luz de métodos de análisis más actuales.

De este modo, en torno a la PWBS-E aquí estudiada, lo mencionado previamente es de suma importancia, ya que los estudios basados en la versión en español hallados en Latinoamérica, y México en particular, con muestras de adolescentes, adultos o adultos mayores, presenta evidencia contradictoria en cuanto a los hallazgos. Tal es así que difieren en cuanto al número de factores que constituyen la escala, los cuales además están sustentados en una gran diversidad de métodos de análisis que en ocasiones presentan fuertes limitaciones que hacen cuestionable su uso. Asimismo, existen reportes que indican la presencia de dos, cuatro, cinco y seis factores, incluso de seis factores de primer orden con uno o dos factores de segundo orden; es decir, mientras que algunos estudios obtienen una estructura similar a la original otros han obtenido resultados distintos.

En este orden de ideas, y considerando la importancia que tiene el BP en el mejor entendimiento de la salud, el bienestar en adultos y, particularmente, en el desarrollo integral del estudiante universitario y la utilidad de su evaluación, fue necesario reanalizar la PWBS a fin de comprobar que cuenta con las bondades psicométricas necesarias para garantizar la validez de la interpretación de sus puntuaciones desde una perspectiva multidimensional (Ryff \& Keyes, 1995) dado su impacto y uso en otros contextos (Ryff, 2014).

Entonces, en este estudio se encuentra que al analizar la PWBS-E desde diferentes enfoques analítico-factoriales (CFA, ESEM y EFA), se afirma su carácter de medida unidimensional. Los primeros análisis no lograron recuperar de forma satisfactoria la estructura de seis factores, evidenciando ítems poco representativos o complejos e incluso factores inexistentes; asimismo, las soluciones factoriales de cinco o cuatro factores tampoco tuvieron éxito. Adicionalmente, se encontraron elevadas correlaciones interfactoriales, así como malas especificaciones asociadas a cargas cruzadas, las cuales imposibilitaban la interpretación de cada dimensión de forma independiente. En vista de ello, se analizaron los ítems de la PWBS-E desde una óptica exploratoria, logrando obtener una medida breve y empíricamente unidimensional.

Otro aspecto relevante a mencionar, y no investigado anteriormente con respecto al PWBS, es la presencia de un factor de método asociado a los ítems invertidos en los modelos multidimensionales evaluados. Es decir, la mayor parte de ítems invertidos producen varianza irrelevante al constructo evaluado -no aportan insumos para la explicación del constructo BP-. Por ello, la interpretación de puntuaciones que usan esos ítems podría estar sesgada en los otros estudios revisados. 
Para terminar, los coeficientes de confiabilidad hallados, tanto de puntuaciones como de constructo, fueron elevados. Esto indica que el error de medición que contempla esta nueva versión es bajo, lo que permitiría usar las puntuaciones para decisiones individuales (e.g., clasificación según datos normativos) e inclusive como medida de eficacia de intervenciones, previo cumplimiento del requisito de invarianza longitudinal y evaluación del error transitorio (Brown, 2015; Dominguez-Lara, 2018).

Desde un punto de vista teórico, es importante resaltar que si no se cuenta con un instrumento que sea capaz de replicar empíricamente lo referido por el enfoque de Ryff (BP como constructo multidimensional), tendría escaso valor para brindar información sobre las dimensiones originales. Por ello, podría entenderse al BP como un constructo que, si bien tiene facetas, es esencialmente unidimensional como ya se pudo apreciar en otros contextos (Chen, Jing, Hayes, \& Lee, 2013). Otra perspectiva de la unidimensionalidad del bienestar es la aportada por Keyes et al. (2008) quien define al bienestar como una evaluación global acerca de la experiencia subjetiva del individuo que incluye el aspecto emocional y social, es decir, un conjunto de experiencias dentro de las cuales cada una es una manifestación de buena calidad de vida.

Entonces, en términos prácticos, disponer de una versión breve y unidimensional de 19 ítems de la PSWB-E favorece la factibilidad por su aplicación de forma rápida (Carvajal, Centeno, Watson, Martínez, \& Sanz-Rubiales, 2011) en un grupo poblacional que puede mostrar apatía ante escalas largas, disminuyendo de esta forma la proporción de no respuesta y aumentando la confiabilidad de las mismas. Por otra parte, la identificación de un único factor facilita la comprensión para quienes no se encuentran muy familiarizados con el uso de escalas psicométricas, potenciando de esta forma su uso tanto en la clínica como en la salud pública (Carvajal et al., 2011). De este modo, tendría aplicaciones en entornos relevantes, tales como la prevención primaria, considerando la vasta evidencia de la relación del BP con conductas de salud (Edmondson \& MacLeod, 2015; Rand et al., 2017; Ridner et al., 2016; Schnettler et al., 2015; Tomba et al., 2014), así como con respecto a la evaluación antes y después de programas de intervención orientados a mejorar el BP de los participantes (Weiss, Westerhof, \& Bohlmeijer, 2016).

En otro orden de ideas, el estudio presentó algunas limitaciones. Por ejemplo, el análisis psicométrico realizado contempla solo estudiantes universitarios, pero el estudio original (Ryff, 1989) comparó adultos jóvenes (18 - 29 años) de mediana edad (30 - 64 años) y adultos mayores (65 años o más) lo que permitió determinar perfiles de edad para las dimensiones del instrumento (e.g., el dominio ambiental y la autonomía se incrementan desde la edad adulta temprana hasta la mediana edad). Otro aspecto a destacar es la ausencia de un reporte de invarianza de medición entre varones y mujeres, ya que si bien existen estudios que comparan esos dos grupos, no existe evidencia de que la medición sea equivalente entre estos. Además, implementar este análisis tiene más sentido cuando el instrumento posee una estructura interna definida que no es el caso del PWBS, donde no existe consenso respecto a su estructura y los métodos predominantes son cuestionables (Dominguez-Lara \& Navarro-Loli, 2018)3/4, por lo que es necesario establecer como línea base una estructura y en estudios posteriores planificar estudios de invarianza. Del mismo modo, el estudio estuvo centrado en la estructura interna de la PWBS$\mathrm{E}$, por lo que no fueron consideradas otras fuentes de evidencias de validez, como la relación con otras variables. Por último, si bien esta versión breve no informa sobre las facetas del BP, se puede seguir una pauta genérica de interpretación basada en su definición (Ryff, 1989), ya que si las dimensiones están superpuestas, es irrelevante interpretarlas por separado porque brindan prácticamente la misma información (Dominguez-Lara \& Navarro-Loli, 2018).

En vista de las limitaciones antes mencionadas, es conveniente ampliar la muestra a otros grupos etarios, 
así como analizar la asociación de la PWBS con otras variables para ver cómo se comporta el constructo BP en relación con otros aspectos psicológicos relevantes para el individuo. Asimismo, otro aspecto relevante que por motivos de extensión del manuscrito no fue desarrollado y que se recomienda para futuros reportes es la elaboración de datos normativos (baremos) que permitan clasificar a las personas por niveles.

En conclusión, una estructura breve y unidimensional de la PWBS-E es la que recibe mayor respaldo empírico presentando indicadores aceptables en lo que concierne a su estructura interna $\mathrm{y}$ confiabilidad.

\section{Conflicto de intereses}

Los autores declaramos no tener conflicto de intereses.

\section{Responsabilidad ética}

El protocolo de investigación fue revisado y aprobado por un comité de investigaciones asignando el número de registro DGI40500201891. Los participantes en el estudio fueron orientados sobre los fines del mismo y firmaron un consentimiento informado. La confidencialidad de los datos fue garantizada.

\section{Contribución de autoría:}

SDL: análisis estadísticos, interpretación de los datos y redacción del manuscrito.

TRG: discusión y revisión final del manuscrito.

CPE: proceso de recolección de la información, revisión final de manuscrito.

ABE: proceso de recolección de la información, revisión final de manuscrito.

EDT: proceso de recolección de la información, revisión final de manuscrito.

YCU: concepción y diseño del estudio, participación en la elaboración del artículo.

\section{Referencias}

Aboalshamat, K., Hou, X. Y., \& Strodl, E. (2015). Psychological well-being status among medical and dental students in Makkah, Saudi Arabia: A crosssectional study. Medical Teacher, 37(1), S75-S81. doi: 10.3109/0142159X.2015.1006612

Asparouhov, T., \& Muthen, B. (2006). Robust Chi Square Difference Testing with Mean and Variance Adjusted Test Statistics. Mplus Web Notes, 10. Recuperado de https://www.statmodel.com/download/webnotes/ webnote10.pdf

Asparouhov, T., \& Muthen, B. (2009). Exploratory Structural Equation Modeling. Structural Equation Modeling: A Multidisciplinary Journal, 16(3), 397438. doi: 10.1080/10705510903008204

Ato, M., López, J. J., \& Benavente, A. (2013). Un sistema de clasificación de los diseños de investigación en psicología. Anales de Psicología, 29(3), 1038-1059. doi: 10.6018/analesps.29.3.178511

Balcázar, P., Esparza, O., \& Gurrola, G. (2018). Psychometric Analysis of the Ryff's Psychological Well-Being Scales in Students of Four Mexican Universities. Psychology, 9(13), 2685-2708. doi: 10.4236/psych.2018.913154

Bhullar, N., Hine, D. W., \& Phillips, W. J. (2014). Profiles of psychological well-being in a sample of Australian university students. International Journal of Psychology, 49(4), 288-294. doi: 10.1002/ijo p.12022

Boehm, J. K., Soo, J., Chen, Y., Zevon, E. S., Hernandez, R., Lloyd-Jones, D., \& Kubzansky, L. D. (2017). Psychological Well-being's Link with Cardiovascular Health in Older Adults. American Journal of Preventive Medicine, 53(6), 791-798. doi: 10.1016/ j.amepre.2017.06.028

Brown, T. (2015). Confirmatory Factor Analysis for Applied Research ( $2^{\mathrm{a}}$ ed.). New York: The Guilford Press.

Byrne, B. (2008). Testing for multigroup equivalence of a measuring instrument: A walk through the process. Psicothema, 20(4), 872-882. Recuperado de http:// www.psicothema.com/pdf/3569.pdf 
Carvajal, A., Centeno, C., Watson, R., Martínez, M., \& Sanz-Rubiales, Á. (2011). ¿Cómo validar un instrumento de medida de la salud? Anales del Sistema Sanitario de Navarra, 34(1), 63-72.

Chen, F. F. (2007). Sensitivity of Goodness of Fit Indexes to Lack of Measurement Invariance. Structural Equation Modeling: A Multidisciplinary Journal, 14(3), 464-504. doi: 10.1080/10705510701301834

Chen, F. F., Jing, Y., Hayes, A., \& Lee, J. (2013). Two Concepts or Two Approaches? A Bifactor Analysis of Psychological and Subjective Well-Being. Journal of Happiness Studies, 14(3), 1033-1068. doi: 10.1007/ s10902-012-9367-X

Chitgian-Urzúa, V., Urzúa, A., \& Vera-Villarroel, P. (2013). Análisis preliminar de las escalas de bienestar psicológico en población chilena. Revista Argentina de Clínica Psicológica, 22(1), 5-14. Recuperado de https://www.redalyc.org/articulo.oa?id=28193 0494002

Cronbach, L. J. (1951). Coefficient alpha and the internal structure of tests. Psychometrika, 16(3), 297-334. doi: 10.1007/BF02310555

Díaz, D., Rodríguez-Carvajal, R., Blanco, A., MorenoJiménez, B., Gallardo, I., Valle, C., \& Dierendonck, D. van (2006). Adaptación española de las Escalas de Bienestar Psicológico de Ryff. Psicothema, 18(3), 572-577. Recuperado de http://www.psicothema.com/ psicothema.asp?id=3255

Dierendonck, D. van (2004). The Construct Validity of Ryff's Scales of Psychological Well-Being and Its Extension with Spiritual Well-Being. Personality and Individual Differences, 36, 629-643. doi: 10.1016/ S0191-8869(03)00122-3

Dierendonck, D. van, Díaz, D., Rodríguez-Carvajal, R., Blanco, A., \& Moreno-Jiménez, B. (2008). Ryff's Six-Factor Model of psychological Well-Being, a Spanish Exploration. Social Indicators Research, 87(3), 473-479. doi: 10.1007/s11205-007-9174-7

Dimitrov, D. M. (2010). Testing for Factorial Invariance in the Context of Construct Validation. Measurement and Evaluation in Counseling and Development, 43(2), 121-149.
DiStefano, C., Liu, J., Jiang, N., \& Shi, D. (2018). Examination of the Weighted Root Mean Square Residual: Evidence for Trustworthiness? Structural Equation Modeling: A Multidisciplinary Journal, 25(3), 453-466. doi: 10.1080/10705511.2017.139 0394

Dominguez-Lara, S. (2018). Invarianza longitudinal y error transitorio de una medida de burnout académico en universitarios. Avaliação Psicológica, 17(3), 311320. doi: 10.15689/ap.2018.1703.14421.04

Dominguez-Lara, S., \& Merino-Soto, C. (2018). Evaluación de las malas especificaciones en modelos de ecuaciones estructurales. Revista Argentina de Ciencias del Comportamiento, 10(2), 19-24. doi: 10.30882/1852.4206.v10.n2.19595

Dominguez-Lara, S., \& Merino-Soto, C. (2019). Medición con Ítem Único del Agotamiento Emocional Académico en Estudiantes Universitarios Peruanos: Evidencias de Validez y Confiabilidad. Revista Iberoamericana de Diagnóstico y Evaluación - e Avaliação Psicológica, 50(1), 45-56. doi: 10.21865/RIDEP50.1.04

Dominguez-Lara, S., \& Navarro-Loli, J. S. (2018). Revisión de metodologías empleadas en los estudios factoriales de la Escala de Bienestar Psicológico de Ryff (versión en español). Evaluar, 18(2), 17-30. doi: 10.35670/1667-4545.v18.n2.20800

Edmondson, O. J. H., \& MacLeod, A. K. (2015). Psychological Well-Being and Anticipated Positive Personal Events: Their Relationship to Depression. Clinical Psychology \& Psychotherapy, 22(5), 418425. doi: 10.1002/cpp.1911

Fernández-Alonso, R., Suárez-Álvarez, J., \& Muñiz, J. (2012). Imputación de datos perdidos en las evaluaciones diagnósticas educativas. Psicothema, 24(1), 167-175. Recuperado de http://www.psicothe ma.com/psicothema.asp?id=3995

Fleming, J., \& Merino, C. (2005). Medidas de simplicidad y ajuste factorial: un enfoque para la construcción y revisión de escalas derivadas factorialmente. Revista de Psicología, 23(2), 252-266. 
Fornell, C., \& Larcker, D. F. (1981). Evaluationg Structural Equation Models with Unobservable Variables and Measurement Error. Journal of Marketing Research, 18(1), 39-50. doi: 10.1177/00 2224378101800104

Gallardo-Cuadra, I., \& Moyano-Díaz, E. (2012). Análisis psicométrico de las escalas Ryff (versión española) en una muestra de adolescentes chilenos. Universitas Psychologica, 11(3), 931-939. Recuperado de https:/ /www.redalyc.org/articulo.oa?id=64724634021

Hu, L., \& Bentler, P. M. (1998). Fit indices in covariance structure modeling: Sensitivity to underparameterized model misspecification. Psychological Methods, 3(4), 424-453. doi: 10.1037/1082-989X.3.4.424

Hunsley, J., \& Mash, E. J. (2008). Developing criteria for evidence-based assessment: An introduction to assessment that work. In J. Hunsley \& E. J. Mash (Eds.), A Guide to Assessments that Work (pp. 3-14). Oxford: Oxford University Press.

Jöreskog, K. G., \& Sörbom, D. (1993). LISREL 8: Structural Equation Modeling with the SIMPLIS Command Language. Chicago, IL, US: Scientific Software.

Kafka, G. J., \& Kozma, A. (2002). The construct validity of Ryff's scales of psychological well-being (SPWB) and their relationship to measures of subjective wellbeing. Social Indicators Research, 57(2), 171-190. doi: 10.1023/A:1014451725204

Keyes, C. L. M., Wissing, M., Potgieter, J., Temane, M., Kruger, A., \& Rooy, S. van (2008). Evaluation of the Mental Health Continuum-Short Form (MHC-SF) in Setswana-speaking South Africans. Clinical Psychology \& Psychotherapy, 15, 181-192. doi: 10.1002/cpp.572

Kline, R. B. (2016). Principles and Practice of Structural Equation Modeling. New York: The Guilford Press.

Lloret-Segura, S., Ferreres-Traver, A., Hernández-Baeza, A., \& Tomás-Marco, I. (2014). El análisis factorial exploratorio de los ítems: una guía práctica, revisada y actualizada. Anales de Psicología, 30(3), 11511169. doi: 10.6018/analesps.30.3.199361

Loera-Malvaez, N., Balcázar-Nava, P., Trejo-González, L., Gurrola-Peña, G. M., \& Bonilla-Muñoz, M. P. (2008). Adaptación de la Escala de Bienestar Psicológico de
Ryff en adolescentes preuniversitarios. Revista Neurología, Neurocirugía y Psiquiatría, 41(3-4), 90-97. Recuperado de https:/www.medigraphic.com/ cgi-bin/new/resumen.cgi?IDARTICULO=70703

Lorenzo-Seva, U., \& Ferrando, P. J. (2013). FACTOR 9.2 A Comprehensive Program for Fitting Exploratory and Semiconfirmatory Factor Analysis and IRT Models. Applied Psychological Measurement, 37(6), 497-498. doi: 10.1177/0146621613487794

Magallares, A., Benito de Valle, P., Irles, J.A., BolañosRíos, P., \& Jauregui-Lobera, I. (2014). Psychological well-being in a sample of obese patients compared with a control group. Nutrición Hospitalaria, 30(1), 32-36. doi: 10.3305/nh.2014.30.1.7515

Malgady, R. (2007). How skew are psychological data? A standardized index of effect size. The Journal of General Psychology, 134(3), 355-359.

Mardia, K. (1970). Measures of multivariate skewness and kurtosis with applications. Biometrika, 57(3), 519-530.

McDonald, R. P., \& Ho, M. H. R. (2002). Principles and practice in reporting structural equation analyses. Psychological Methods, 7, 64-82. doi: 10.1037/108 2-989X.7.1.64

Medina-Calvillo, M. A., Gutiérrez-Hernández, C. Y., \& Padrós-Blázquez, F. (2013). Propiedades psicométricas de la Escala de Bienestar Psicológico de Ryff en población mexicana. Revista de Educación Desarrollo, 27, 25-30. Recuperado de http://www.cuc s.udg.mx/revistas/edu_desarrollo/anteriores/27/ 027_Padros.pdf

Muthén, L. K., \& Muthén, B. O. (1998-2015). Mplus User's guide ( $7^{\mathrm{a}}$ ed.). Los Angeles, CA: Muthén \& Muthén.

Pendergast, L. L., Embse, N. von der, Kilgus, S. P., \& Eklund, K. R. (2017). Measurement equivalence: A non-technical primer on categorical multi-group confirmatory factor analysis in school psychology. Journal of School Psychology, 60, 65-82. doi: 10.10 16/j.jsp.2016.11.002

Rand, K., Vallis, M., Aston, M., Price, S., Piccinini-Vallis, H., Rehman, L., \& Kirk, S. F. L. (2017). «It is not the diet; it is the mental part we need help with.» A multilevel analysis of psychological, emotional, and 
social well-being in obesity. International Journal of Qualitative Studies on Health and Well-Being, 12(1). doi: 10.1080/17482631.2017.1306421

Reyna, G. V., Delfino, G. I., \& Mele, S. V. (Noviembre, 2013). Estudio psicométrico de la Escala de Bienestar Psicológico en militares argentinos. Trabajo presentado en el V Congreso Internacional de Investigación y Práctica Profesional en Psicología. XX Jornadas de Investigación. Noveno Encuentro de Investigadores en Psicología del MERCOSUR. Facultad de Psicología de la Universidad de Buenos Aires, Argentina.

Ridner, S. L., Newton, K. S., Staten, R. R., Crawford, T. N., \& Hall, L. A. (2016). Predictors of well-being among college students. Journal of American College Health, 64(2), 116-124. doi: 10.1080/074 48481.2015.1085057

Rodríguez, M., \& Ruiz, M. (2008). Atenuación de la asimetría y de la curtosis de las puntuaciones observadas mediante transformaciones de variables: Incidencia sobre la estructura factorial. Psicológica, 29, 205-227.

Rosa-Rodríguez, Y., Negrón-Cartagena, N., MaldonadoPeña, Y., Quiñones-Berrios, A., \& Toledo-Osorio, N. (2015). Dimensiones de bienestar psicológico y apoyo social percibido con relación al sexo y nivel de estudio en universitarios. Avances en Psicología Latinoamericana, 33(1), 31-43. doi: 10.12804/apl3 3.01.2015.03

Ryff, C.D. (1989). Happiness is everything, or is it? Explorations on the meaning of psychological wellbeing. Journal of Personality and Social Psychology, 57, 1069-1081.

Ryff, C. D. (2014). Psychological Well-Being Revisited: Advances in the Science and Practice of Eudaimonia. Psychotherapy and Psychosomatics, 83(1), 10-28. doi: 10.1159/000353263

Ryff, C. D., \& Keyes, C. L. (1995). The structure of psychological well-being revisited. Journal of Personality and Social Psychology, 69(4), 719-727.

Saris, W. E, Satorra, A., \& Veld, W. M. van der (2009). Testing Structural Equation Modeling or Detection of Misspecifications? Structural Equation Modeling, 16, 561-582. doi: 10.1080/10705510903203433
Schnettler, B., Miranda, H., Lobos, G., Orellana, L., Sepulveda, J., Denegri, M., ... Grunert, K. G. (2015). Eating habits and subjective well-being. A typology of students in Chilean state universities. Appetite, 89, 203-214. doi: 10.1016/j.appet.2015.02.008

Shrive, F. M., Stuart, H., Quan, H., \& Ghali, W. A. (2006). Dealing with missing data in a multi-question depression scale: a comparison of imputation methods. BMC Medical Research Methodology, 6(1), 57. doi: 10.1186/1471-2288-6-57

Timmerman, M. E., \& Lorenzo-Seva, U. (2011). Dimensionality Assessment of Ordered Polytomous Items with Parallel Analysis. Psychological Methods, 16, 209-220. doi: 10.1037/a0023353

Tomás, J. M., Meléndez, J. C., Oliver, A., Navarro-Pardo, E., \& Zaragoza, G. (2010). Efectos de método en las escalas de Ryff: Un estudio en población de personas mayores. Psicológica, 31, 383-400.

Tomás, J. M., Sancho, P., Melendez, J. C., \& Mayordomo, T. (2012). Resilience and coping as predictors of general well-being in the elderly: A structural equation modeling approach. Aging \& Mental Health, 16(3), 317-326. doi: 10.1080/13607863.2011.615737

Tomba, E., Offidani, E., Tecuta, L., Schumann, R., \& Ballardini, D. (2014). Psychological well-being in out-patients with eating disorders: a controlled study. International Journal of Eating Disorders, 47(3), 252-258. doi: 10.1002/eat.22197

Véliz-Burgos, A. (2012). Propiedades psicométricas de la Escala de Bienestar Psicológico y estructura factorial en universitarios chilenos. Psicoperspectivas. Individuo y Sociedad, 11(2), 143-163. doi: 10.5027/ psicoperspectivas-Vol11-Issue2- fulltext-196

Watkins, M. W. (2018). Exploratory Factor Analysis: A Guide to Best Practice. Journal of Black Psychology, 44(3), 219-246. doi: 10.1177/0095798418771807

Weiss, L. A., Westerhof, G. J., \& Bohlmeijer, E. T. (2016). Can we increase psychological well-being? The effects of interventions on psychological well-being: A meta-analysis of randomized controlled trials. PloS ONE, 11(6). doi: 10.1371/journal.pone.0158092

West, S. G., Taylor, A. B., \& Wu, W. (2012). Model fit and model selection in structural equation modeling. 
In R. H. Hoyle (Ed.), Handbook of Structural Equation Modeling (pp. 209-231). New York, NY: Guilford.

Yang-Wallentin, F., Jöreskog, K. G., \& Luo, H. (2010). Confirmatory Ordinal Variables With Misspecified Models. Structural Equation Modeling A Factor
Analysis of Multidisciplinary Journal, 17(3), 392423. doi: $10.1080 / 10705511.2010 .489003$

Yu, C., \& Muthen, B. (Abril, 2002). Evaluation of model fit indices for latent variable models with categorical and continuous outcomes. Trabajo presentado en el encuentro anual de la American Educational Research Association, New Orleans, L.A.

\section{Sergio Dominguez-Lara}

Instituto de Investigación de Psicología, Universidad de San Martín de Porres, Lima, Perú.

Doctor en Psicología e investigador en Instituto de Investigación de Psicología (USMP). Interesado en temas vinculados a psicometría y evaluación psicológica.

ORCID: 0000-0002-2083-4278

sdominguezl@usmp.pe

Tania Romo-González

Área de Biología y Salud Integral, Instituto de Investigaciones Biológicas, Universidad Veracruzana, México.

Doctora en Ciencias Biomédicas e Investigadora del Instituto de Investigaciones Biológicas. Miembro del Sistema Nacional de Investigadores, México. Estudia las interacciones entre los subsistemas (inmunológico, nervioso, endocrino, psicológico y social), la salud y el bienestar del individuo.

tromogonzalez@uv.mx

\section{Carolina Palmeros-Exsome}

Facultad de Nutrición, Región Veracruz, Universidad Veracruzana, México.

Doctora en Nutrición y Directora de Facultad de Nutrición, región Veracruz de la Universidad Veracruzana, México. Su trabajo se enfoca en el tema de nutrición y abordaje de la obesidad.

cpalmeros@uv.mx

\section{Antonia Barranca-Enríquez}

Centro de Estudios y Servicios en Salud, Universidad Veracruzana, México.

Doctora en Gobierno y Administración Pública en Psicología y coordinadora del Centro de Estudios y Servicios en Salud, Universidad Veracruzana, México.

Su trabajo se centra en evaluación integral de la salud con enfoque preventivo.

abarranca@uv.mx

Eduardo del Moral-Trinidad

Facultad de Medicina, Región Xalapa, Universidad Veracruzana, México.

Licenciado en Medicina y asistente de investigación en el Instituto de Salud Pública. Ha participado en proyectos sobre estado nutricio, autorregulación y bienestar en estudiantes universitarios.

lalo2226@live.com.mx

\section{Yolanda Campos-Uscanga}

Instituto de Salud Pública, Universidad Veracruzana, México.

Doctora en Psicología e investigadora de tiempo completo del Instituto de Salud Pública. Es miembro del Sistema Nacional de Investigadores en México. Su línea de investigación se enfoca en el bienestar, los estilos de vida saludables y la prevención de la obesidad.

Autor corresponsal: ycampos@uv.mx 


\section{Anexos}

Tabla 6

Análisis factorial confirmatorio de la PWBS-E: muestra de replicación

\begin{tabular}{cc}
\hline & $\lambda$ \\
\hline Ítem 1 & .614 \\
Ítem 3 & .477 \\
Ítem 6 & .627 \\
Ítem 7 & .719 \\
Ítem 11 & .603 \\
Ítem 12 & .742 \\
Ítem 17 & .721 \\
Ítem 18 & .699 \\
Ítem 19 & .752 \\
Ítem 21 & .603 \\
Ítem 23 & .711 \\
Ítem 24 & .752 \\
Ítem 28 & .655 \\
Ítem 31 & .833 \\
Ítem 32 & .518 \\
Ítem 35 & .547 \\
Ítem 37 & .746 \\
Ítem 38 & .733 \\
Ítem 39 & .525 \\
\hline
\end{tabular}

Nota: $\lambda_{=}$carga factorial.

Tabla 7

Análisis de invarianza de medición entre muestras de calibración y replicación

\begin{tabular}{lcccccc}
\hline & CFI & RMSEA (IC 90\%) & $\chi^{2}(\mathrm{gl})$ & $\Delta$ CFI & $\Delta$ RMSEA & $\Delta \chi^{2}(\mathrm{gl})$ \\
\hline Configural & .919 & $.101(.097, .104)$ & $2925.619(304)^{* * *}$ & - & - & - \\
Métrica & .956 & $.072(.069, .076)$ & $1759.323(322)^{* * *}$ & .037 & -.029 & $21.486^{\text {ns }}(18)$ \\
Fuerte & .958 & $.062(.059, .065)$ & $1770.250(416)^{* * *}$ & .002 & -.010 & $85.398^{\text {ns }}(94)$ \\
\hline
\end{tabular}

Nota: $\mathrm{IC}=$ intervalo de confianza; $\mathrm{gl}=$ grados de libertad; $* * *=p<.001 ;{ }^{\mathrm{ns}}=p>.05 ; \mathrm{ME}=$ Modelo estructural . 